\title{
Study of comparing cutting force signal features for dry, air cooling and minimum quantity lubrication (MQL) drilling
}

\author{
Jie XU*, Keiji YAMADA**, Katsuhiko SEKIYA**, Ryutaro TANAKA** and Yasuo YAMANE** \\ *Jiangsu key laboratory of advanced food manufacturing equipment and technology, \\ 1800 Lihu Avenue, Jiangnan University, Wuxi, China \\ E-mail: xujie@jiangnan.edu.cn \\ **Graduate school of Engineering, Hiroshima University, Hiroshima, Japan
}

Received: 30 September 2016; Revised: 13 January 2017; Accepted: 7 June 2017

\begin{abstract}
Applying Minimum Quantity Lubrication (MQL) for machining processes such as drilling can obtain process performance improvement comparing with completely dry situations, and reduce the use of machining fluids considerably comparing with flood cooling. Although many researches have been reported on the comparison of temperature, tool wear, surface roughness and chip patterns between dry and MQL cutting, relatively fewer literatures about the cutting forces and their features can be found. In this paper, first dry and MQL drilling of cast iron, S45C, SUS304 and Ti alloy were conducted, and then life term of dry, air cooling and MQL drilling of SUS304 were carried out. TiCN coated HSS drill bits with double inner coolant holes were applied to produce air cooling and MQL internally. The thrust force and torque were monitored by a dynamometer and, time, frequency, and time-frequency domain features of them are extracted and compared. Especially there are some newly generated features derived from transforming the thrust force and torque to two orthogonal forces and further being analyzed in a rectangular coordinate. Experiment results show that, for different workpiece materials MQL affects different force features in different ways and for SUS304 tool life is greatly lengthened, friction is suppressed, chip jamming is alleviated and both the static and dynamic components of cutting forces reduce when air cooling and MQL are applied. Moreover, it is revealed that the differences of features between dry and other two conditions are quite obvious but those between air cooling and MQL are inconspicuous.
\end{abstract}

Key words : MQL, Drilling, Cutting forces, Signal features, Stainless steel

\section{Introduction}

Machining with coolants and lubricants can obtain improvement, increase productivity, and extend tool life by reducing tool wear. Nevertheless, the use of cutting fluids in machining processes has been questioned because they do give rise to certain problems such as the high cost of coolant and its disposal fee, threats to the operators' health and pollution to the environment (Sreejith and Ngoi, 2000). Therefore, recent research in industry and academia has sought ways to reduce the use of machining fluids (Meena and El Mansori, 2011). The most logical way to eliminate the drawbacks caused by the using of cooling lubricant is dry machining. But in most cases dry machining cannot guarantee the same performance as using cooling lubricant under same conditions. In many machining operations, minimum quantity lubrication (MQL) is the key to successful dry machining (Weinert et al., 2004). The MQL is generally defined as feeding the fluid media (oil, water etc.) to the cutting point in tiny quantities and usually with the assistance of transport medium, e.g., air (Dhar et al., 2006).

Many literatures on researches about comparing the performance of drilling under MQL to other conditions such as dry, air cooling, flooded emulsion can be found.

Bhowmick and Alpas compared drilling of aluminum-silicon alloys (319 $\mathrm{Al})$ under dry, water MQL $\left(\mathrm{H}_{2} \mathrm{O}\right.$ spray at rate of $30 \mathrm{ml} / \mathrm{h}$ externally) and flooded $\left(\mathrm{H}_{2} \mathrm{O}\right.$ at $\left.30 \mathrm{l} / \mathrm{h}\right)$ conditions (Bhowmick and Alpas, 2008). And it was observed that, compared to dry drilling lower torque and thrust forces were required under $\mathrm{H}_{2} \mathrm{O}-\mathrm{MQL}$, and the adhesion and the sudden increase of torque were alleviated. They also conducted drilling experiments of cast magnesium alloys (AM60) under dry, MQL (water and fatty acid based as lubricant at $10 \mathrm{ml} / \mathrm{h}$ externally) and flooded (mineral oil) conditions (Bhowmick et al., 2010), and it was found that, comparing dry with MQL the average torque and thrust forces and the maximum temperature generated in the workpiece reduced to the level similar to flooded drilling while the tool life was 
lengthened greatly. And they also studied the role of non-hydrogenated diamond like carbon (NH-DLC) coated drills on minimum quantity lubrication drilling of magnesium alloys (AZ91) (Bhowmick and Alpas, 2011), and it was found that with the application of MQL $\left(\mathrm{H}_{2} \mathrm{O}\right.$ at rate of $\left.30 \mathrm{ml} / \mathrm{h}\right)$, tool life was increased and, drill torque as well as the temperature was reduced to levels comparable to the conventional flooded drilling using mineral oil.

Heinemann et al compared the differences of torque under dry and MQL (synthetic ester at $18 \mathrm{ml} / \mathrm{h}$ rate) conditions on the of small twist drills in deep hole drilling (Heinemann et al., 2006). The data showed that the torque curves under dry and MQL are distinctly different, first was in the initial penetration period where increase of torque for MQL was lower due to lower friction between tool and workpiece, second was the obvious increase gradient of torque for dry caused by the increased heat and friction and third was more fluctuations of torque for dry attributed to a discontinuous chip motion through the non-lubricated chip flute.

Tasdelen et al compared the wear, chip contact, forces/torques and surface finish of short hole drilling tests under dry compressed air, MQL at different oil amounts $(5 / 15 / 23 \mathrm{ml} / \mathrm{h})$ and emulsion with indexable inserts (Tasdelen et al., 2008). The results showed that MQL and compressed air usage have resulted lower wear compared to emulsion, cutting with compressed air resulted in side flow and sticking of the work piece material on the walls of the hole leading to bad surface finish, and different cooling lubrication conditions produced different cutting force variation patterns.

Zeilmann and Weingaertner found that the measured temperatures with application of MQL internally through the tool were 50\% lower than those obtained with MQL applied with an external nozzle, and with the increase of the temperature, smaller feed forces were measured for the smaller resistance of the material to the penetration of the cutting edge while this tendency did not occur for the torque (Zeilmann and Weingaertner, 2006).

However, there is few intensive research on the cutting forces generated during drilling under different cooling/lubrication conditions can be found, which may concern the analysis of the features of the cutting forces that could indicate different performances. The authors (Xu et al., 2014) have found that some specially defined features have very strong correlations to the drill corner wear. Therefore, the main goal of this work is to make a comparison of features extracted from cutting forces, thrust force and torque specifically, generated during drilling different workpiece materials and under different cooling and lubrication conditions such as air cooling, MQL and dry.

\section{Methodology}

\subsection{Features of cutting force signals}

In this paper, features extracted from the thrust force and torque are defined and categorized in three domains: time, frequency and time-frequency domains as summarized by Teti et al (Teti et al., 2010). But what is novel here is that, there are some newly defined features derived from transforming thrust force $(F z)$ and torque $(M z)$ to two orthogonal forces, equivalent thrust force $(F t)$ and equivalent principal force $(F p)$, and further analyzing them in a rectangular coordinate together with their resultant force $(F r)$. All the features are defined as listed in table 1 and the detailed definition could be found in (Xu et al., 2014).

Table 1 the classification of force features

\begin{tabular}{|c|c|c|}
\hline Categories & Subgroups & Features \\
\hline \multirow[b]{2}{*}{$\begin{array}{l}\text { Time } \\
\text { domain }\end{array}$} & Static & $F t_{-} M, F p_{-} M, \rho_{-} M, \theta_{-} M, F t_{-} R M S, F p_{-} R M S, \rho_{-} R M S, \theta_{-} R M S$ \\
\hline & Dynamic & $\begin{array}{l}F t_{-} R N G, F p_{-} R N G, \rho_{-} R N G, \theta_{-} R N G, F t_{-} S T D, F p_{-} S T D \\
F r_{-} S T D, F t_{-} V e l, F p_{-} V e l, F r_{-} V e l, F r_{-} C H A\end{array}$ \\
\hline \multirow{3}{*}{$\begin{array}{l}\text { Frequency } \\
\text { domain }\end{array}$} & $F t(0-1 \mathrm{kHz})$ & $F t_{-} P S D 1 \_M, F t_{-} P S D 1 \_R N G, F t_{-} P S D 1 \_R M S, F t \_P S D 1 \_S T D$ \\
\hline & $F p(0-1 \mathrm{kHz})$ & $\begin{array}{l}F p_{-} P S D 1 \_M, F p \_P S D 1 \_R N G, F p_{-} P S D 1 \_R M S, \\
F p_{-} P S D 1 \_S T D\end{array}$ \\
\hline & $F p(5-6 \mathrm{kHz})$ & $\begin{array}{l}F p_{2} P S D 2 \_M, F p_{-} P S D 2 \_R N G, F p_{-} P S D 2 \_R M S, \\
F p_{-} P S D 2 \_S T D\end{array}$ \\
\hline $\begin{array}{l}\text { Time- } \\
\text { frequency } \\
\text { domain }\end{array}$ & $\begin{array}{l}F B i \text { : the } i \text {-th sub } \\
\text { frequency band }\end{array}$ & $\begin{array}{l}F t_{-} F B i_{2} R N G, F p_{-} F B i_{-} R N G, \rho_{-} F B i_{-} R N G, \theta_{-} F B i_{-} R N G, \\
F t_{-} F B i_{-} S T D, F p_{-} F B i_{-} S T D, F r_{-} F B i_{-} S T D, F t_{-} F B i_{-} V e l, \\
F p_{-} F B i_{-} V e l, F r_{-} F B i_{-} V e l, F p_{-} F B i_{-} C H A\end{array}$ \\
\hline
\end{tabular}

As shown in table 1, in this paper, features are expressed in the form of parameters with suffixes. The “_M" suffix denotes the mean value of the parameter, and the “_ $R M S$ ” means the root mean square, and the “_RNG” as the range, 
and "_STD" as standard deviation. The "_PSDI" and "_PSD2" indicate the power spectral density values of the parameter in frequency range 1 and 2, which are specified in 2.2.3. The "_FBi" means the feature value is generated from the reconstructed sub-signal of the $i$-th frequency band as expressed in 2.2.4. The "_Vel" and "_CHA" are two specially defined features which means the forces trajectory point average moving "velocity" and the convex hull area of the distributed $\mathrm{Fr}$ trajectory points as shown in Fig. 2. Expressions of $F t_{-} V e l, F p_{-} V e l$ and $F r_{-} V e l$ are as follows:

$$
\begin{aligned}
& F t_{-} V e l=\frac{1}{t_{0}} \sum_{i=2}^{m}\left|F t_{i}-F t_{i-1}\right| \\
& F p_{-} V e l=\frac{1}{t_{0}} \sum_{j=2}^{m}\left|F p_{j}-F t_{j-1}\right| \\
& F r_{-} V e l=\frac{1}{t_{0}} \sum_{k=2}^{m} \sqrt{\left(F t_{k}-F t_{k-1}\right)^{2}+\left(F p_{k}-F p_{k-1}\right)^{2}}
\end{aligned}
$$

where $t_{0}$ means the sampling time, $m$ indicates the total sampling number, and $F t_{i}$ and $F p_{j}$ represent the $i$-th and $j$ th sampling value of $F t$ and $F p$.

\subsection{Signal analysis}

\subsubsection{The segmentation of signals according cutting stages}

The whole drilling process of a single workpiece can be divided into 3 stages, according to the cutting edges position. Forces increase when the cutting edges enter but not entirely inside the work piece, and usually this is called entry stage. And forces decrease when cutting edges penetrate workpiece and this is commonly named penetration stage. To obtain force features under a relatively stable condition, data intercepted during the stage between the former two stages named medial stage is used for further analysis as demonstrated in Fig. 1.

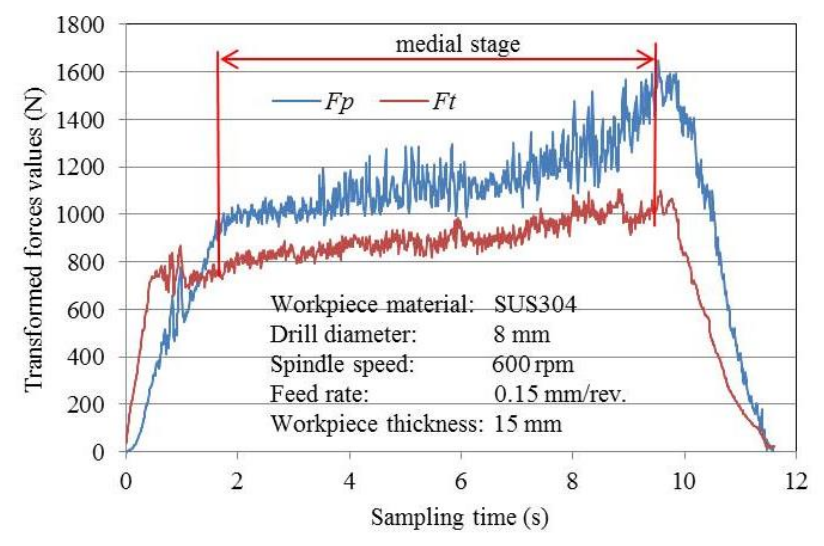

Fig. 1 The intercepted segment of cutting forces

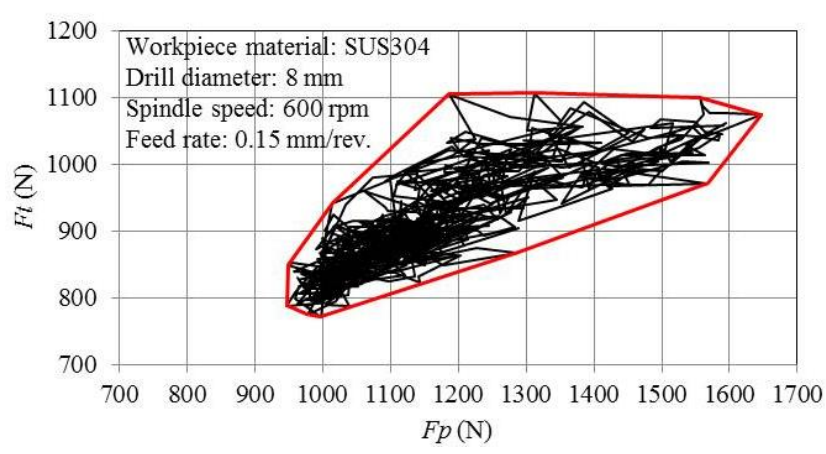

Fig. 2 Plot of $F t$ to $F p$ in a rectangular coordinate and the convex hull

\subsubsection{Time domain force signals}

Then the intercepted force signals can be plotted in a rectangular coordinate as shown in Fig. 2 and used for the extraction of time domain features. The convex hull of the resultant force trajectory data points is illustrated by red lines.

\subsubsection{Frequency domain force signals}

The sampling frequency applied is $20 \mathrm{kHz}$ and the time duration is 8 seconds for this condition, therefore according to the sampling theorem, the analyzable frequency range is $0-10 \mathrm{kHz}$ with $0.25 \mathrm{~Hz}$ resolution. Fig. 3 shows the logarithmic scale PSD of $F t$ and $F p$, where we can find that both $F t$ and $F p$ have higher PSD at low frequency range $(0-1000 \mathrm{~Hz})$ and the PSD of $F p$ has a peak around $5550 \mathrm{~Hz}$ which is actually the natural frequency of the workpiece fixture system. And further investigation in the near natural frequency range $(5450-5650 \mathrm{~Hz})$ shows that the PSD of $F p$ under dry condition is much higher than that under MQL as shown in Fig. 4. 


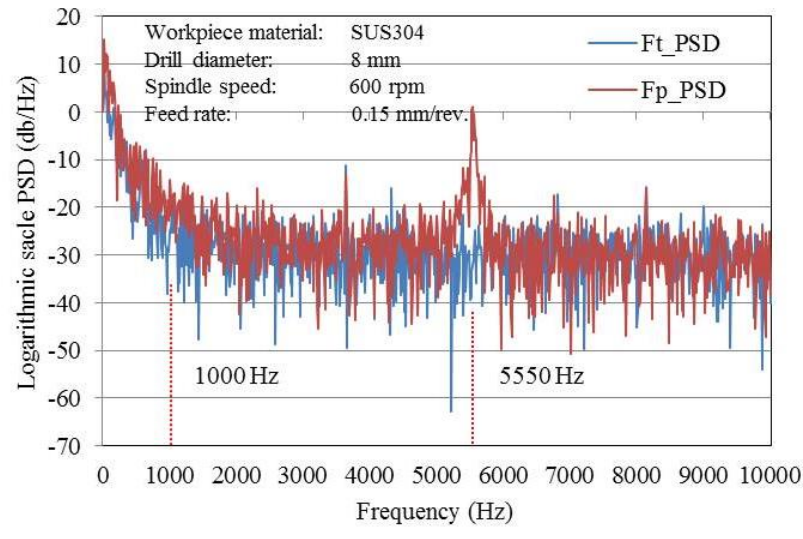

Fig. 3 Frequency domain force signals

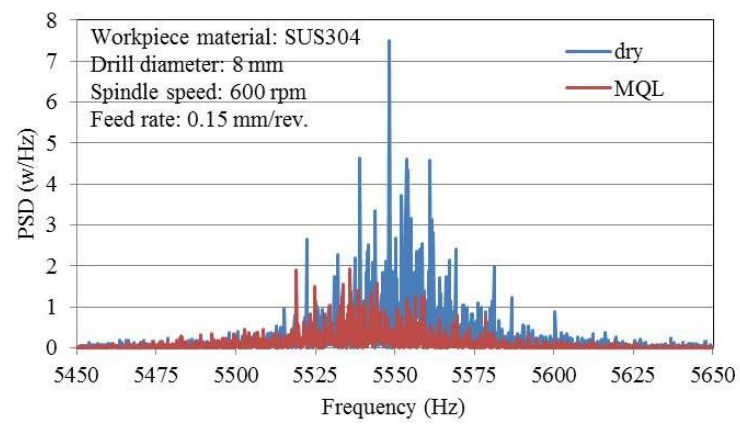

Fig. 4 Comparison of PSD of Fp under dry and MQL in near natural frequency range

\subsubsection{Time-frequency domain signals}

The WPT conducted was 3 leveled dividing the full analyzable frequency band $(0-10 \mathrm{kHz})$ into 8 sub frequency bands varying from $1-1.25 \mathrm{kHz}$ to $8.75-10 \mathrm{kHz}$, in consideration of keeping natural frequency $(5550 \mathrm{~Hz})$ in center of a sub frequency band (specifically 5-th sub frequency band, 5-6.25 kHz) and the computing complexity. Fig. 5 shows the reconstructed $F t$ to $F p$ from this very sub frequency band under dry and MQL conditions.

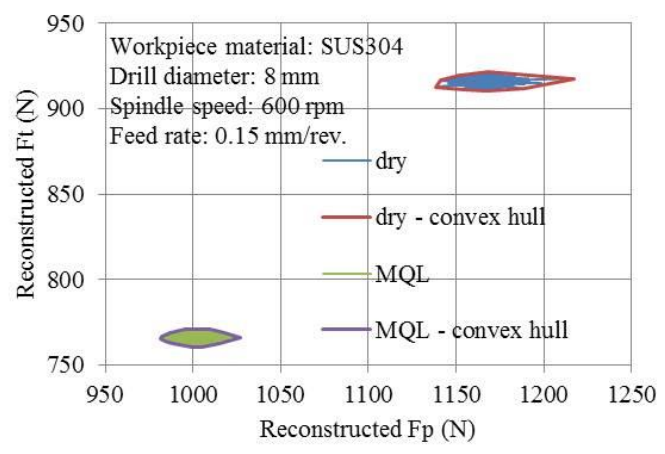

Fig. 5 Reconstructed $F t$ to $F p$ from 5 -ith sub frequency band $(5-6.25 \mathrm{kHz})$ under dry and MQL conditions

We can find from Fig. 5 that, firstly the reconstructed resultant force trajectory of dry drilling has higher average Ft and Fp than that of MQL drilling for its upper right position; secondly the convex hull area, i.e. the distribution area of the reconstructed resultant force trajectory of dry drilling is larger than that of MQL drilling signifying higher fluctuation; thirdly the reconstructed resultant force trajectory data points under MQL are more concentrated than that under dry condition due to less sudden increase of Fp. Consequently, the sub frequency band (5-6.25 kHz) is discussed in this paper.

\section{Experimental setup and procedure}

The experiments were carried out in a Mitsubishi MPA V45 vertical CNC machining center with 10 HP of power and $6000 \mathrm{rpm}$ rated speed. Since there is no through coolant inside the spindle, the MQL was supplied through a coolant inducer (BT40-OSL25-165, BIG DAISHOWA SEIKI CO., LTD.). The air flow from the compressor was regulated to $0.4 \mathrm{MPa}$ and imported into a MQL applicator (FK2-S1-LM, FUJI BC ENGINEERING CO., LTD.) to actuate the oil pump. The MQL oil applied was LB-1 also supplied from FUJI BC. The lubricant oil specifications are shown in table 2. The air flow and oil were transported through a pipe-in-pipe tube, with the oil in the inner pipe, and they were mixed inside the chamber of the coolant inducer till reaching. The lubricant quantity was set at $30 \mathrm{ml} / \mathrm{h}$. The drills applied were $8 \mathrm{~mm}$ HSS TiCN coated twist drills with 2 inner coolant holes (VP-HO-GDS 8594580, CO., LTD.), and its dimensions are shown in Fig. 6. The air flow only or mixture with the oil lubricant was finally jetted to the cutting area internally through the drill bit. The overall experimental setup is shown in Fig. 7. 
Table 2 lubricant oil specifications

\begin{tabular}{l|l}
\hline Specific gravity $\left(\mathrm{g} / \mathrm{cm}^{3}, 20^{\circ} \mathrm{C}\right)$ & 0.92 \\
\hline Flash point $\left({ }^{\circ} \mathrm{C}\right)$ & 320 \\
\hline Viscosity $\left(\mathrm{mm}^{2} / \mathrm{s}, 40^{\circ} \mathrm{C}\right)$ & 37 \\
\hline Pour point $\left({ }^{\circ} \mathrm{C}\right)$ & $-20^{\circ} \mathrm{C}$ \\
\hline
\end{tabular}

Fig. 6 Drill bit dimensions

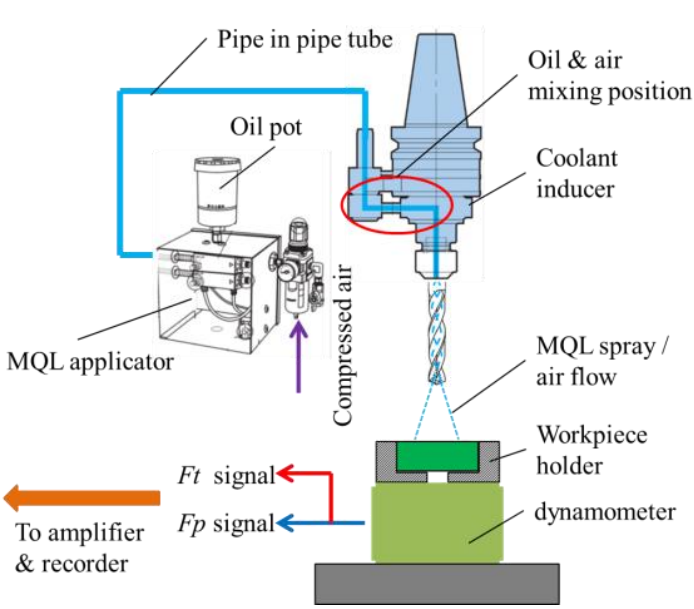

Fig. 7 Experimental setup

Workpieces were cylindrically shaped, and fixed inside a specially made workpiece holder, which was then mounted on top of a dynamometer. The cutting forces, thrust force and torque, were sampled, amplified and recorded by a Kistler 9365B dual channel dynamometer, a Kistler 5073 charge amplifier (Frequency response $20 \mathrm{kHz}$ ) and a Yokogawa DL750 scope recorder. The signal sampling frequency was $20 \mathrm{kHz}$, and the acquired digital data was then analyzed on a computer.

The experiments proceed as follows: first, 4 different workpiece materials, cast iron, low carbon steel (S45C), stainless steel (SUS304) and Ti alloy ( $\alpha-\beta$ Ti6Al4V), were applied and both dry and MQL drilling were conducted for 3 times for each material; second, SUS304 were selected and tool life tested under different cooling/lubrication conditions. The reason of choosing SUS304 was discussed in the next section.

\section{Results and discussions}

As list in table 1, there are 42 features totally, a considerable amount of them. And since the experiments under same conditions were repeated for several times, the feature numbers proliferate again, thus a comparison strategy is extremely necessary. In this paper, a measuring indicator "difference ratio $(D R)$ " is introduced to give a relatively more direct and clear comparing result.

The difference ratio of one specific feature between two different cooling and lubrication conditions for several experimental repetitions is defined as:

$$
\begin{aligned}
& D R=\frac{\overline{F V_{C O N 1}}-\overline{F V_{C O N 2}}}{\overline{F V_{C O N 2}}} \times 100 \% \\
& \overline{F V_{C O N 1}}=\frac{\sum_{p=1}^{T} F V_{C O N 1}(p)}{T} \\
& \overline{F V_{C O N 2}}=\frac{\sum_{q=1}^{U} F V_{C O N 2}(q)}{U}
\end{aligned}
$$

In equation (5) and (6), $F V_{C O N 1}(p)$ and $F V_{C O N 2}(q)$ are respectively the feature value under condition 1 , for instance dry, of the p-th repeated experiment and feature value under condition 2, let's say MQL, of the q-th repeated experiment, $\overline{F V_{C O N 1}}$ and $\overline{F V_{C O N 1}}$ are the averaged feature values, and $\mathrm{T}$ and $\mathrm{U}$ are the total repeated times of experiments under these two conditions. We can see from equation (4) that the difference ratio of one feature means how much percentage is the average feature value under condition 1 higher than that under condition 2 , that is, the higher the difference ratio value the greater different between these two conditions for this specific feature, that is the better feature.

More strictly, each feature value of condition 1 should be higher than any value of this feature of condition 2 or lower without exception. However, this criterion cannot be satisfied for all features. When comparing $\mathrm{T}$ and $\mathrm{U}$ experimental repetitions under condition 1 and 2, there are $\mathrm{T} \times \mathrm{U}$ comparing pairs, then for each pair, use $F V_{C O N 1}(p)$ subtract $F V_{C O N 2}(q)$ and if the result has same positive negative characteristic as the $D R$, add 1 for the other indicator --- criterion satisfied count (CSC). The criterion satisfied count (CSC) can be defined as: 


$$
C S C=\left\{\begin{array}{l}
\operatorname{count}\left(F V_{C O N 1}(p) \geq F V_{C O N 2}(q)\right), D R \geq 0 \\
\operatorname{count}\left(F V_{C O N 1}(p)<F V_{C O N 2}(q)\right), D R<0
\end{array} p=1, \ldots, T ; q=1, \ldots, U\right.
$$

where count(criterion) means the total count when the criterion is satisfied.

Thus apparently, the $D R$ indicator concerns more about the general average differences between two conditions while the CSC indicator involves the repeatability of the differences shown by the features.

\subsection{Drilling of 4 materials under dry and MQL}

The drilling experiments were conducted with $0.15 \mathrm{~mm} / \mathrm{rev}$. feed rate, and $38,28,12,6 \mathrm{~m} / \mathrm{s}$ cutting speed for cast iron, S45C, SUS304 and Ti alloy respectively, indicated by CI, SC, SU and Ti in the figures. The drill depth for cast iron and S45C is $20 \mathrm{~mm}$, and for SUS304 and Ti alloy is $10 \mathrm{~mm}$ to prevent major wear. For each material, both the dry and MQL drilling were triplicated, thus $T=3$ and $U=3$ for CSC in Equation (7).

\subsubsection{Time domain features}

The trajectories of the resultant force $(F r)$ of the 4 materials under dry and MQL are illustrated in the rectangular coordinate composed of $F p$ and $F t$ in Fig. 8. As shown in the figure, MQL decreases the cutting forces for other materials but $\mathrm{S} 45 \mathrm{C}$. The difference ratios of time domain static features for the 4 materials are shown in Fig. 9. Positive $D R$ values mean that the averaged features under dry is higher than under MQL and negative ones on the contrary. And moreover, the $D R$ is indicated as a solid bar when the CSC equals the possible maximum value, and it is denoted as slash pattern filled bar if lower with the CSC labeled, similarly hereinafter. The highest CSC here is 9 for both dry and MQL drilling being repeated for 3 times. From Fig. 9 we can find that, for cast iron, the $F t \_M$ feature can tell the difference between dry and MQL very clearly, which is about 13\% higher for dry than MQL; for S45C, all 4 features differ slightly between dry and MQL and the $F t_{-} M$ is higher for MQL probably due to less heat and higher hardness; for SUS304, Fp_ $M$ and $\rho_{-} M$ are better than $F t_{-} M$ and $\theta_{-} M$ for higher $D R$ and $C S C$; and for Ti alloy, higher $\theta_{-} M$ of MQL than dry shows that $F p_{-} M$ decreases more than $F t_{-} M$. And it shows that for all of these materials, $F p \_M$ decreases due to less friction between drill, chip and workpiece.

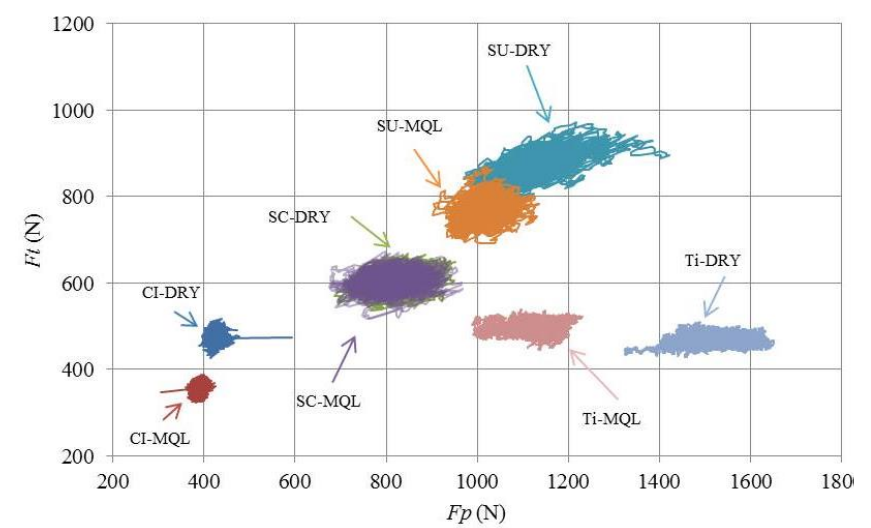

Fig. 8 Trajectories of the time domain resultant force $(F r)$ in the $F p$-Ft rectangular coordinate

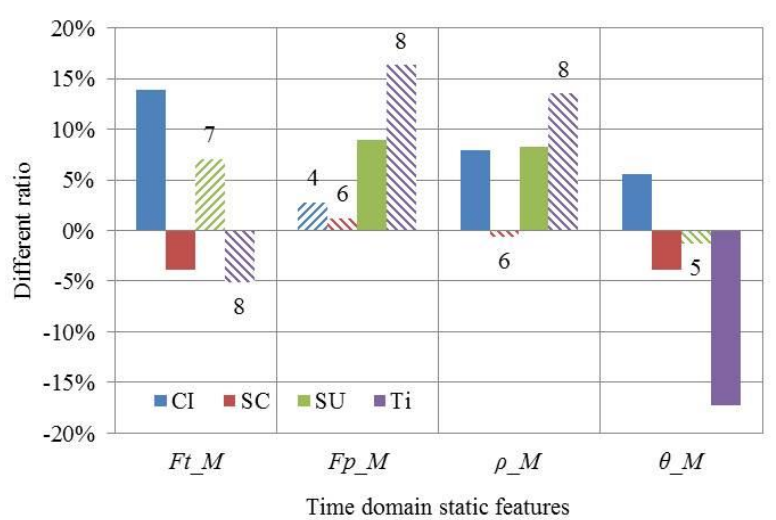

Fig. $9 D R$ and $C S C$ of time domain static features for the 4 materials between dry and MQL drilling

Fig. 10 shows the $D R$ and $C S C$ of time domain dynamic features between dry and MQL for 4 materials. We can find that most of the time domain dynamic features have relatively higher DR and CSC 9 for SUS304, especially for $F r_{-} C H A$, which under dry is more than $60 \%$ higher than under MQL. This result implies that the utility of MQL is more effective for drilling SUS304 measured by time dynamic features, and this is the reason for using SUS304 in the second stage experiments. For cast iron, it is shown that the $F p_{-} R N G, F p_{-} S T D$ and $F p_{-} V E L$ increase with the MQL adoption, meaning the fluctuation of torque increases, opposite to the average feature $F p_{-} M$ which decreases as shown in Fig. 8 . The average hole wall roughness under dry drilling of cast iron is $R a=3.63 \mathrm{um}$, which is obtained by measuring each workpiece for 5 times at 5 equal partitions of the circle and $2.5 \mathrm{~mm}$ beneath the top surface, while for MQL drilling the value is $R a=2.4 \mathrm{um}$. When drilling cast iron, the chips are discontinuous and tiny; and when the MQL is applied, the 
air flow blows up the chips inside the bored hole and the viscous lubricant tend to stick the tiny chips to the hole wall and fill the valleys, therefore, both higher dynamic of torque and lower roughness can be obtained.

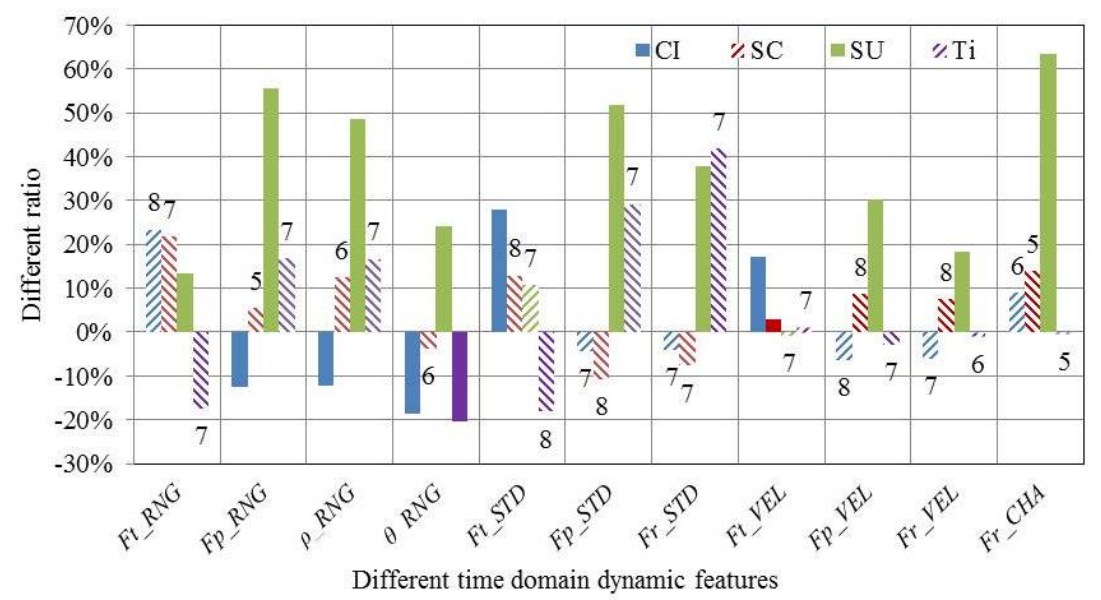

Fig. $10 D R$ and CSC of time domain dynamic features between dry and MQL for 4 materials

\subsubsection{Frequency domain features of $F t(0-1 \mathrm{kHz})$}

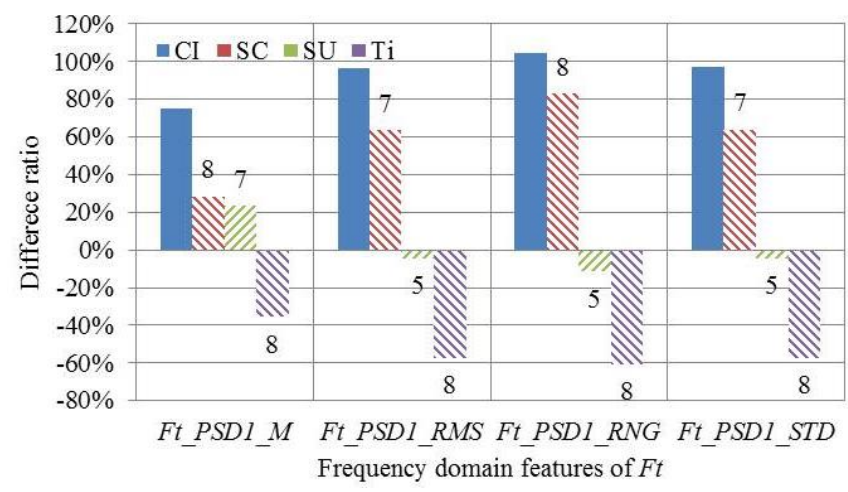

Fig. $11 D R$ and $C S C$ of $F t$ PSD1 features between dry and MQL for 4 materials

As shown in Fig. 11, Ft frequency domain features of cast iron perform better than other materials, implying the possibility of applying these features as indicators for MQL usage. And very little difference is shown for SUS304.

\subsubsection{Frequency domain features of $F p(0-1 \mathrm{kHz})$}

The DRs of Fp PSD1 features are much higher for SUS304 and Ti alloy, as we can see in Fig. 12, where it shows the $D R$ of $F p_{-} P S D 1 \_R N G$ for Ti alloy even is about $250 \%$. Unlike what's shown in Fig. 11 , the $D R$ s of $F p$ PSD1 features are lower for cast iron and S45C. Moreover, we can find that Ft PSD1 features of S45C are higher for dry than MQL but $F p$ PSD1 features are lower for dry than MQL, which means the lubrication can reduce the thrust force while the cooling effect may increase the torque for S45C. And Ti alloy on the contrary, the lubrication effect for rotation direction and cooling for axis direction are more dominant. The difference between $\mathrm{S} 45 \mathrm{C}$ and $\mathrm{Ti}$ alloy shows that it is more difficult for the lubricant reaching the tool top zone for Ti alloy, and the temperature difference between dry and MQL in drill top zone where most thrust force being generated is higher than that in cutting edge zones where most torque being generated. The temperature at the rake face corner of dill bit under dry and MQL was detected and compared (Le Coz et al., 2012) and the temperature of workpiece near the hole wall under MQL drilling was measured (Zeilmann and Weingaertner, 2006). However, unfortunately there is no direct comparison of the difference of temperature for dry and MQL between these two zones. 


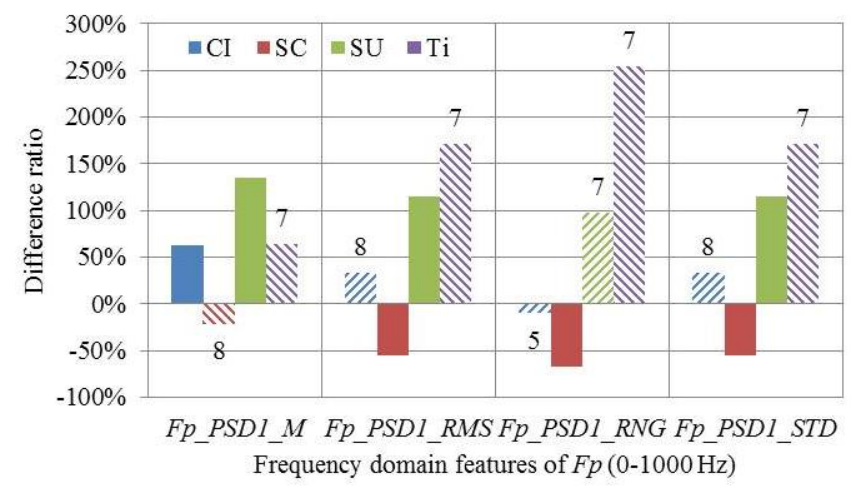

Fig. $12 D R$ and CSC of Fp PSD1 features between dry and MQL for 4 materials

\subsubsection{Frequency domain features of $F p(5-6 \mathrm{kHz})$}

The $D R$ and $C S C$ of $F p$ PSD2 features between dry and MQL for 4 materials are demonstrated in Fig. 13. It shows that SUS304 is the one makes highest difference between dry and MQL for these features. The fluctuation of Fp in this frequency range is caused by the vibration excited by the friction between the chips and hole wall, thus lower feature values with MQL means the friction has been suppressed due to better chip evacuation.

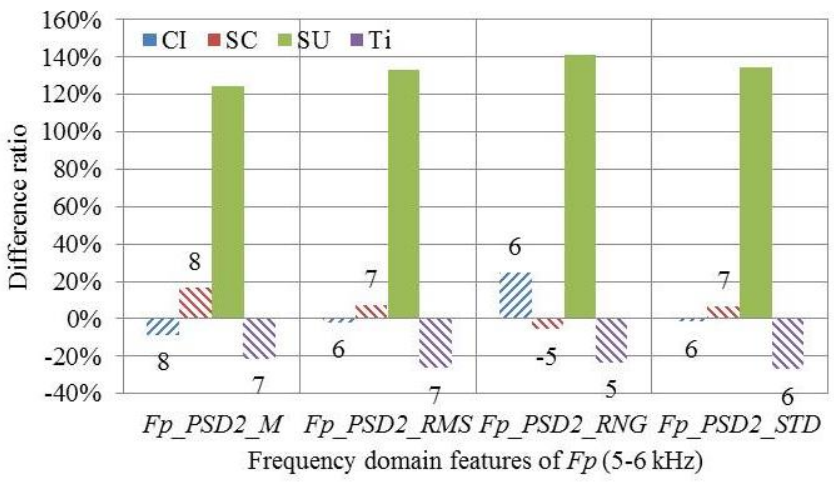

Fig. $13 D R$ and $C S C$ of $F p$ PSD2 features between dry and MQL for 4 materials

\subsubsection{Time-frequency domain features}

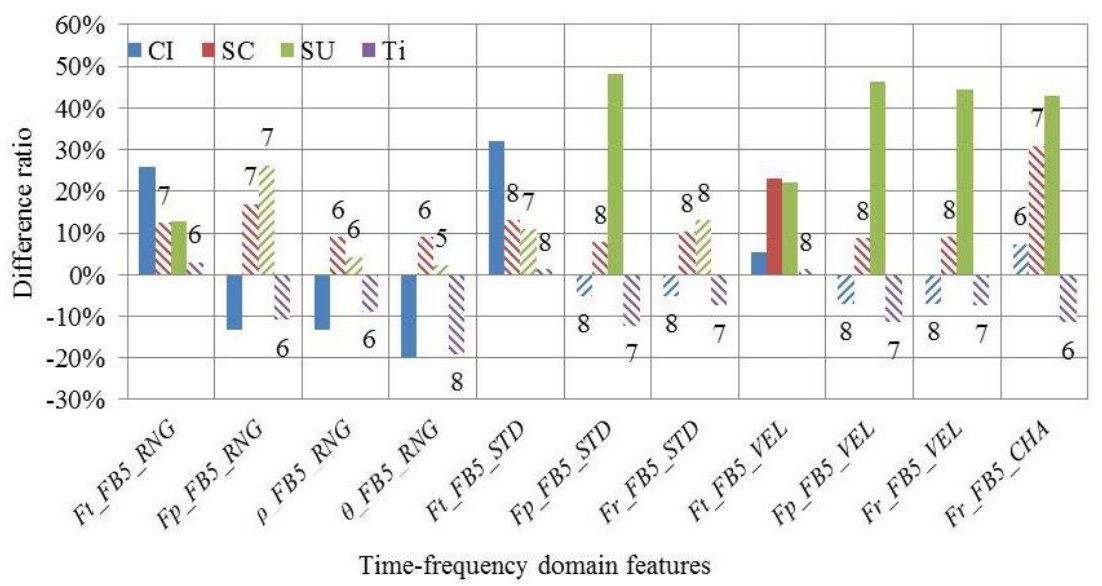

Fig. $14 D R$ and $C S C$ of time-frequency features between dry and MQL for 4 materials

The DR and CSC of time-frequency features between dry and MQL for 4 materials are shown in Fig. 14. For cast iron, as discussed before, the vibration of Fp is higher for MQL than dry, and we can confirm from Fig. 12 and Fig. 13 that this augment mainly takes place in the frequency range near natural frequency while not in the lower frequency range $(0-1 \mathrm{kHz})$ as shown in Fig. 12 which says the average PSD in this frequency range of dry is higher than MQL. For 
S45C and SUS304, all features have positive values meaning MQL decreases the vibration of forces in this frequency range.

\subsection{Drilling of SUS304 under dry, air cooling and MQL}

The dry, air cooling and MQL (represented as DRY, AIR and MQL hereinafter) drilling tests for SUS304 were experimented with $15 \mathrm{~m} / \mathrm{s}$ cutting speed and $0.15 \mathrm{~mm} / \mathrm{rev}$. feed rate. The workpieces were prepared with a $15 \mathrm{~mm}$ thickness because pretests showed that higher thickness easily cause tool breakage under dry condition resulting in an extremely short tool life.

\subsubsection{Comparison of wear process}

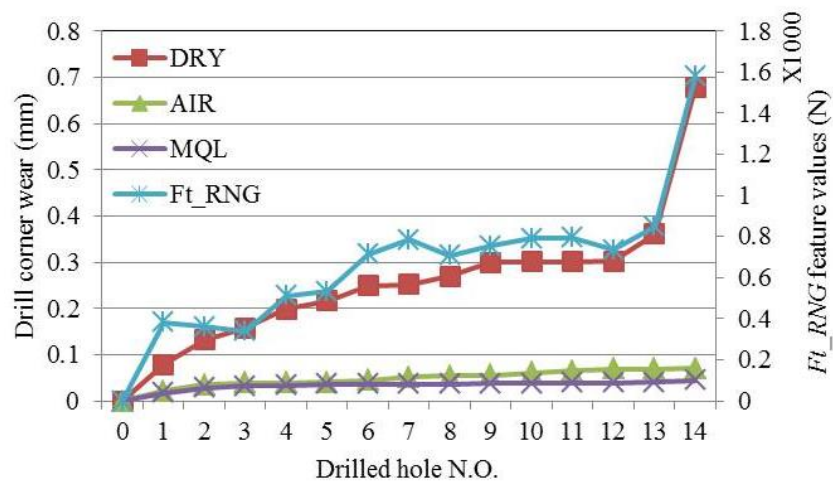

Fig. 15 Drill corner wear measure after each hole under dry, air cooling and MQL

As shown in Fig. 15, the drill worn very quickly during dry drilling and served for 14 holes in all with the 13-th and 14-th hole as the last tool life stage. The wear proceeded much more slowly with air cooling and MQL, and lowest wear rate was obtained with MQL. Therefore, the data acquired from the $1^{\text {st }}$ to $12^{\text {th }}$ hole of these three conditions were used for comparison. The $F t_{-} R N G$ feature values for the 14 holes experiments are also shown in Fig. 15. We can find that the $F t_{-} R N G$ feature values keep pace with the increase of corner wear very well, providing a 0.978 correlation. Therefore, $F t_{-} R N G$ could be applied as an indicator for drill wear prediction.

\subsubsection{Comparison of hole wall surface roughness}

The bored hole wall roughness was measure for each workpiece under three conditions, which is shown in Fig. 16. There is no big difference between air cooling, MQL and dry with acceptable corner wear (less than $0.3 \mathrm{~mm}$ ). But the side wall roughness increases significantly for the $13^{\text {th }}$ and $14^{\text {th }}$ hole for which the drill corner are $0.361 \mathrm{~mm}$ and 0.677 $\mathrm{mm}$. for these two holes, it can be observed clearly that there is much workpiece material being stuck on the side wall.

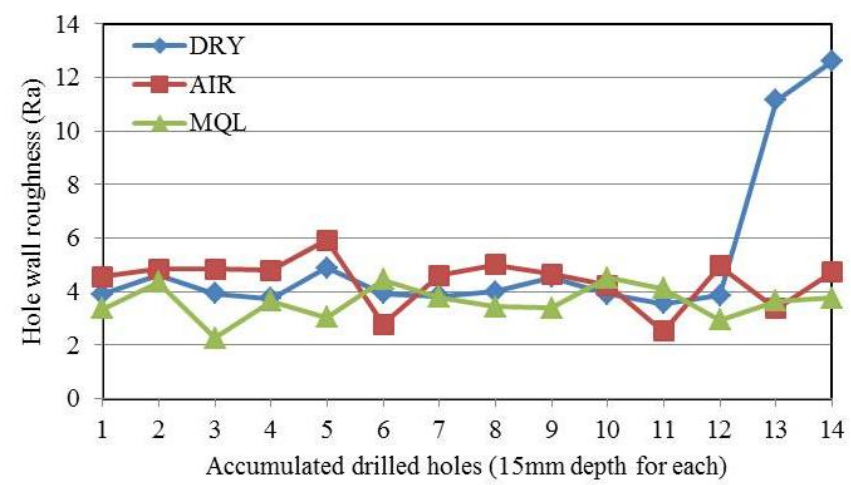

Fig. 16 Hole wall surface roughness ( $\mathrm{Ra}$ ) for dry, air cooling and MQL 


\subsubsection{Comparison of force features}

On account of both cooling and lubrication effect which could be obtained by MQL, feature forces with MQL are expected to be the lowest among these three conditions, with dry as the highest and air cooling in the middle. Therefore, comparing between the three conditions was conducted as pairs of DRY-MQL, DRY-AIR and AIR-MQL. And for some features, the $D R$ between DRY-MQL and DRY-AIR is much higher than that between AIR-MQL, thus the DR between AIR-MQL is plotted as the secondary axis. Since for each condition, 12 experimental data sets were used, the highest $C S C$ is 144 .

\subsubsection{Time domain features}

As shown in Fig. 17, all time domain static features have positive value of $D R$ between DRY-MQL and DRY-AIR, which means that both air cooling and MQL can reduce the power consumption when drilling SUS304. However, the difference between AIR and MQL are not so distinct for time domain features, of which the DRs are lower than 5\% and CSCs are all lower than 144.

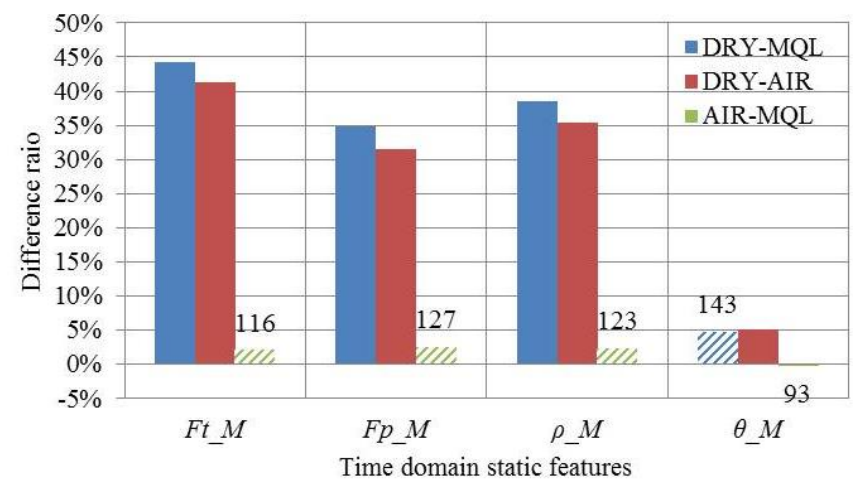

Fig. $17 D R$ and CSC of time domain static features between DRY-MQL, DRY-AIR and AIR-MQL

The difference between DRY-MQL and DRY-AIR are more obvious for the time domain dynamic features as shown in Fig. 18, but still between AIR and MQL there is no big difference. The $D R$ of the $F r_{-} C H A$ feature has the highest value about $500 \%$, and then the standard deviation features near $280 \%$, the range features close to $180 \%$ and velocity features $20 \%$ approximately. The CSCs of all these features between DRY-MQL and DRY-AIR are equal to the maximum value 144, which means significant difference of dynamic components of cutting forces between dry drilling and drilling with cooling and lubrication.

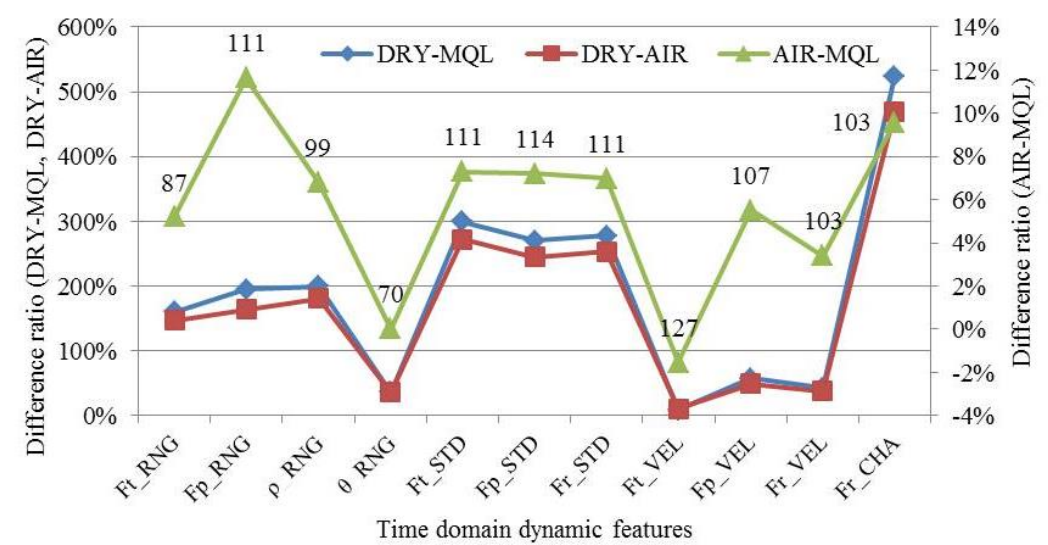

Fig. $18 D R$ and CSC of time domain dynamic features between DRY-MQL, DRY-AIR and AIR-MQL

\subsubsection{Frequency domain features}

As shown in Fig. 19 and Fig. 20, in the lower frequency range 0-1 kHz, features of both $F t$ and $F p$ have extremely high DRs between DRY-MQL and DRY-AIR, which means that vibrations of forces in this frequency range are excessive. And comparing to the result shown in Fig. 11and Fig. 12, we can find that the drill wear have seriously 
affected the comparison results for these features. The $D R$ s of frequency domain features in low frequency range between AIR and MQL increase to the range about $15 \%$ to $40 \%$, which is higher than of time domain static features (lower than 5\%) and time domain dynamic features (lower than 10\%).

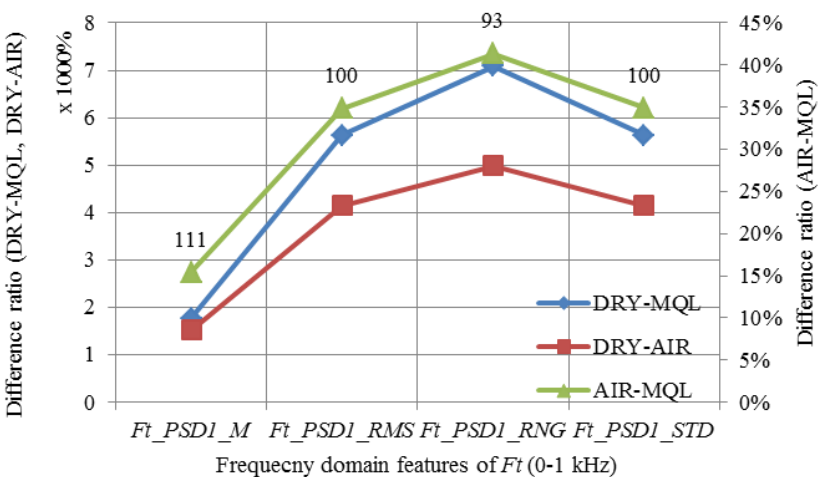

Fig. $19 D R$ and $C S C$ of frequency domain features of $F t$ (0-1 kHz) between DRY-MQL, DRY-AIR and AIR-MQL

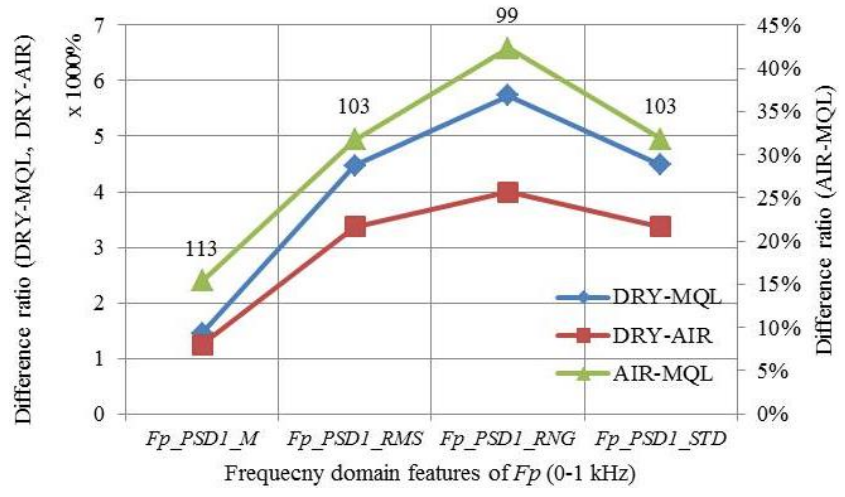

Fig. $20 D R$ and $C S C$ of frequency domain features of $F p$ $(0-1 \mathrm{kHz})$ between DRY-MQL, DRY-AIR and AIR-MQL

As shown in Fig. 21, although the $D R$ s of $F p$ frequency features in 5-6 kHz range are an order of magnitude lower than that in $0-1 \mathrm{kHz}$ range, they are still much higher than the time domain features. However, there is no big chance between these two frequency ranges for Fp frequency features of AIR and MQL.

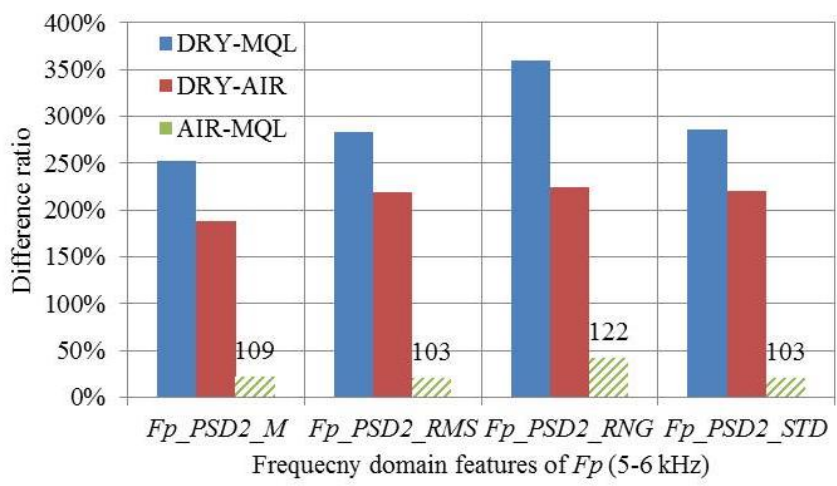

Fig. $21 D R$ and $C S C$ of frequency domain features of Fp (5-6 kHz) between DRY-MQL, DRY-AIR and AIR-MQL

\subsubsection{Time-frequency domain features}

Finally in Fig. 22, we may find that the convex hull area features and range features have higher $D R$ values than standard deviation features and velocity features for DRY-MQL and DRY-AIR, however lower CSC values. Similar result can be found for AIR-MQL, but still no feature reaches the maximum CSC value.

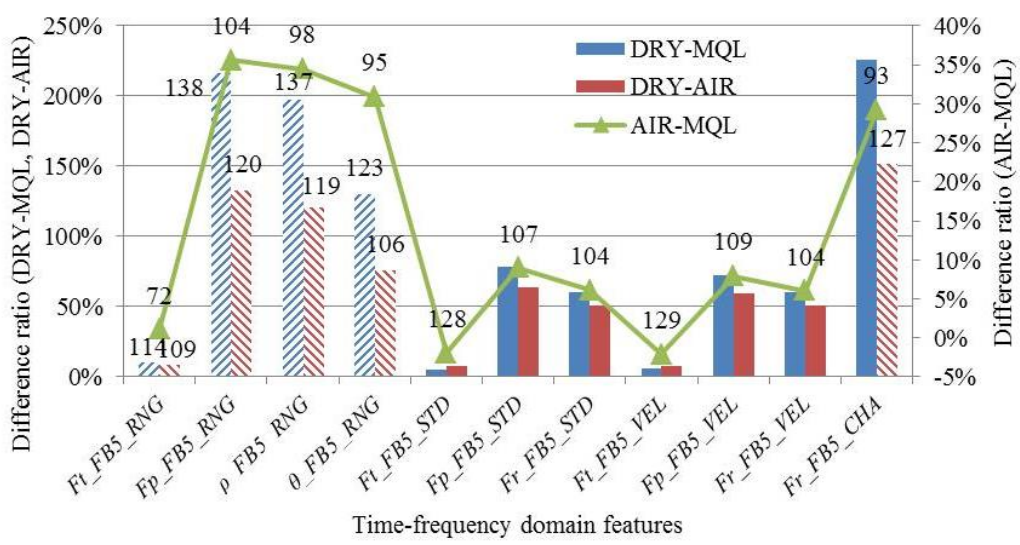

Fig. $22 D R$ and $C S C$ of time-frequency domain features between DRY-MQL, DRY-AIR and AIR-MQL 


\section{Conclusions}

This paper presented a comparison of cutting forces in the manner of comparing the force features between dry, air cooling and MQL drilling. These features are generally defined and extracted from time, frequency and time-frequency domains, and some novel features are newly defined such as the velocity features and the convex hull area feature. To evaluate the differences of numerous features between different conditions, the difference ratio $(D R)$ and criterion satisfied count $(C S C)$ are proposed to present more direct and clear result. Based on the findings from the drilling experiments under conditions specified in this paper, the followings can be concluded:

(1) For different workpiece materials MQL affects different force features in different ways.

(2) As MQL is applied, the static component of thrust force decreases and dynamic component of torque increases for cast iron; the dynamic component of torque in the low frequency range increases; both static and dynamic components of thrust force and torque decrease for SUS304; and comparing to thrust force the average torque decrease much more for Ti alloy.

(3) For SUS304, tool life is greatly lengthened, friction is suppressed, chip jamming is alleviated and both the static and dynamic components of cutting forces reduce when air cooling and MQL are applied, but not better surface finish.

(4) The features differences between dry and air cooling, dry and MQL are quite obvious, and those between air cooling and MQL are inconspicuous.

\section{Acknowledgments}

This work is supported by the Natural Science Foundation of Jiangsu China (Grant No. BK20160182) and the Fundamental Research Funds from Jiangnan University.

\section{References}

Bhowmick, Sukanta, and A. T. Alpas, Minimum quantity lubrication drilling of aluminium-silicon alloys in water using diamond-like carbon coated drills, International Journal of Machine Tools \& Manufacture, Vol. 48, No. 1213(2008), pp1429-1443, DOI:10.1016/j.ijmachtools.2008.04.010

Bhowmick, Sukanta, M. J. Lukitsch, and A. T. Alpas, Dry and minimum quantity lubrication drilling of cast magnesium alloy (AM60), International Journal of Machine Tools \& Manufacture, Vol. 50, No. 5(2010),pp444-457

Bhowmick, S., and A. T. Alpas, The role of diamond-like carbon coated drills on minimum quantity lubrication drilling of magnesium alloys, Surface \& Coatings Technology, Vol. 205, No. 23(2011), pp5302-5311

Dhar, N. R., M. Kamruzzaman, and M. Ahmed, Effect of minimum quantity lubrication (MQL) on tool wear and surface roughness in turning AISI-4340 steel, Journal of Materials Processing Technology, Vol. 172, No. 2(2006), pp299-304, DOI:10.1016/j.jmatprotec.2005.09.022

Heinemann, R., et al., Effect of MQL on the tool life of small twist drills in deep-hole drilling, International Journal of Machine Tools \& Manufacture, Vol 46, No. 1(2006), pp1-6, DOI:10.1016/j.ijmachtools.2005.04.003

Le Coz, G., et al., Measuring temperature of rotating cutting tools: Application to MQL drilling and dry milling of aerospace alloys, Applied Thermal Engineering, Vol 36, No.1 (2012), pp434-441,

Meena, A., and M. E. Mansori, Study of dry and minimum quantity lubrication drilling of novel austempered ductile iron (ADI) for automotive applications, Wear, Vol. 271, No. 9-10 (2011), pp2412-2416, DOI:10.1016/j.wear. 2010.12.022

Sreejith, P. S, and B. K. A. Ngoi, Dry machining: Machining of the future, Journal of Materials Processing Technology, Vol. 101, No. 1-3 (2000), pp287-291, DOI:10.1016/S0924-0136(00)00445-3

Tasdelen, B., T. Wikblom, and S. Ekered, Studies on minimum quantity lubrication (MQL) and air cooling at drilling, Journal of Materials Processing Technology, Vol. 200, No. 1-3(2008), pp339-346

Teti, R., et al., Advanced monitoring of machining operations, CIRP Annals - Manufacturing Technology, Vol 59, No.2(2010), pp717-739, DOI:10.1016/j.cirp.2010.05.010

Weinert, K., et al, Dry Machining and Minimum Quantity Lubrication, CIRP Annals - Manufacturing Technology, Vol 53, No. 2(2004), pp511-537, DOI:10.1016/S0007-8506(07)60027-4

$\mathrm{Xu}$, Jie, et al., Effect of different features to drill-wear prediction with back propagation neural network, Precision Engineering, Vol. 38, No. 4(2014), pp791-798, DOI:10.1016/j.precisioneng.2014.04.007

Zeilmann, Rodrigo Panosso, and W. L. Weingaertner, Analysis of temperature during drilling of Ti6Al4V with minimal quantity of lubricant, Journal of Materials Processing Technology, Vol. 179, No. 1-3(2006), pp124-127, DOI:10.1016/j.jmatprotec.2006.03.077 